\title{
ON DIFFERENTIAL RINGS OF ENTIRE FUNCTIONS
}

\author{
BY
}

\section{A. H. CAYFORD AND E. G. STRAUS( $\left.{ }^{1}\right)$}

ABSTRACT. Consider an entire function $f$ which is a solution of the differential equation

$$
\left[c_{0}(z)+c_{1}(z) D+\ldots+c_{m}(z) D^{m}\right]\left(f^{n}\right)=P\left(f, f^{\prime}, \ldots, f^{(k)}\right)
$$

where $c_{i}(z)$ are entire functions in a differential ring $R$ and $P$ is a polynomial in a differential field related to $R$. We prove the following

THEOREM. If $f$ satisfies the equation above then $f$ is of finite type in case $R=\mathrm{C}$ and of finite exponential order in case $R=\mathrm{C}[z]$.

We use this result to prove a conjecture made in [2] that entire functions of order $\rho<s$, all of whose derivatives at $s$ points are integers in an imaginary quadratic number field, must be solutions of linear differential equations with constant coefficients and therefore of order $\leqslant 1$.

1. Introduction. In this paper we consider entire functions $f(z)$ which satisfy differential equations of the form

$$
L\left(f^{n}\right)=P\left(f, f^{\prime}, \ldots, f^{(k)}\right)
$$

where $L$ is a linear differential operator

$$
L=c_{0}(z)+c_{1}(z) D+\ldots+c_{m}(z) D^{m}
$$

with $c_{i}(z)$ entire functions in a differential ring $R$ and $P\left(x_{0}, x_{1}, \ldots, x_{k}\right)$ a polynomial in $F\left[x_{1}, \ldots, x_{k}\right]$ where $F$ is a differential field of meromorphic functions related to $R$ in the manner described below, and $\operatorname{deg} P<n$.

1.2. Definition. $A$ growth ring $M_{R}$ of a ring of entire functions $R$ is a semiring (closed under addition and multiplication) of functions $M(r)$ so that for each $g \in R$ there exists an $M(r) \in M_{R}$ such that

Received by the editors December 27, 1973 and, in revised form, May 17, 1974.

AMS (MOS) subject classifications (1970). Primary 30A64, 30A66; Secondary 30A62, 30A68, 30A70, 30A82, 34A20, 34A45.

Key words and phrases. Integer valued entire function, linear differential operator, approximation by algebraic integers, growth rate.

(1) Work of the first author supported in part by the National Research Council of Canada; work of the second author supported in part by NSF Grant GP 28696. 


$$
M(r, g)=\max _{|z|=r}|g(z)| \leqslant M(r) ; \quad 0 \leqslant r<\infty .
$$

A growth ring $M_{F}$ for a field of meromorphic functions $F$ is a semiring of functions $M(r)$ so that for every $h \in F$ there exist entire functions $h_{1}, h_{2}$ and a function $M(r) \in M_{F}$ so that $h=h_{1} / h_{2}$ and $\max \left\{M\left(r, h_{1}\right), M\left(r, h_{2}\right)\right\} \leqslant M(r)$, $0 \leqslant r<\infty$.

We now assume that the field $F$ above is related to $R$ by having a growth ring

$$
M_{F}=\left\{\sum_{i=1}^{N} C_{i 1} \exp \left(C_{i 2} r M_{i}(r)\right) \mid M_{i}(r) \in M_{R}, C_{i 1}, C_{i 2}>0\right\}
$$

In $\S 3$ we shall see that all entire functions $h$ which satisfy a homogeneous linear differential equation with coefficients in $R$ do have an upper bound in $M_{F}$ defined by (1.3).

Our main result is the following.

1.4. THEOREM. If $f$ satisfies (1.1) and $R$ is either $\mathrm{C}$, the ring of complex constants, or $\mathrm{C}[z]$, the ring of polynomials, then $M(r, f)$ has an upper bound in $M_{F}$. That is, $f$ is of finite exponential type in case $R=\mathbf{C}$ and of finite exponential order in case $R=\mathrm{C}[z]$.

In $\S 2$ we prove Theorem 1.4 in the case $R=\mathbf{C}$, and apply the result to prove a conjecture stated by one of us [2] concerning integer valued entire functions. In particular we prove that entire functions of order $\rho<s$, all of whose derivatives at $s$ points are integers in an imaginary quadratic number field, must be solutions of linear differential equations with constant coefficients and therefore satisfy $\rho \leqslant 1$. The result in $\S 2$ has also been used by one of us [4] to characterize completely those differential rings, $R$, of meromorphic functions which are finite dimensional modules over the ring of linear differential operators with constant coefficients. That is, there exists an $n$ so that for any $n+1$ functions $f_{0}, f_{1}, \ldots, f_{n}$ in $R$ there exist linear differential operators with constant coefficients $L_{0}, L_{1}, \ldots, L_{n}$ not all 0 so that $L_{0} f_{0}+L_{1} f_{1}+\ldots+$ $L_{n} f_{n}=0$.

In $\S 3$ we prove the case of Theorem 1.4 in which $R=\mathbf{C}[z]$, the ring of polynomials, to indicate the general method. In $\S 4$ we state some proved and conjectured additional results.

2. The case $R=\mathrm{C}$ and applications to the arithmetic of entire functions. Since the operator $L$ in (1.1) is in $C[D]$ we may assume without loss of generality that $c_{m}=1$ and hence 


$$
L=(D-\alpha) \cdots\left(D-\alpha_{m}\right)
$$

If we set $g=P\left(f, f^{\prime}, \ldots, f^{(k)}\right)$ we get

$$
f^{n}=L^{-1} g=h+\exp \left(\alpha_{1} z\right) \int_{0}^{z} \exp \left(\left(\alpha_{2}-\alpha_{1}\right) z_{1}\right) d z_{1} \int_{0}^{z} \exp \left(\left(\alpha_{3}-\alpha_{2}\right) z_{2}\right) d z_{2}
$$

$$
\cdots \int_{0}^{z_{m-1}} \exp \left(-\alpha_{m} z_{m}\right) g\left(z_{m}\right) d z_{m}
$$

where $L h=0$.

Now we can choose $M_{R}=M_{\mathrm{C}}=R_{+}$, the semiring of positive real numbers. The corresponding $M_{F}$ is $\left\{\Sigma_{i=1}^{n} C_{i 1} \exp \left(C_{i 2} r\right) \mid C_{i j}>0\right\}$. It is clear that $M(r, h)$ has an upper bound in $M_{F}$ and since the coefficients of $P$ are, by hypothesis, meromorphic functions of bounded exponential type, we get $g$ an entire function which can be written in the form $P^{*}\left(f, f^{\prime}, \ldots, f^{(k)}\right) / g_{1}=g^{*} / g_{1}$ where $g_{1}$ is a common denominator for the coefficients of $P$ so that $P^{*}$ has entire coefficients with upper bounds in $M_{F}$, and $M\left(r, g_{1}\right)$ has an upper bound in $M_{F}$.

In order to obtain an estimate for the maximum of $1 / g_{1}$ on a circle of radius $r$ we will use the following minimum modulus theorem due to Levin $[3$, p. 21].

2.2. THEOREM. Let $f(z)$ be holomorphic in the disk $|z| \leqslant 2 e R(R>0)$ with $f(0)=1$ and let $\eta$ be an arbitrary positive number not exceeding $3 e / 2$. Then inside the disk $|z| \leqslant R$, but outside of a family of excluded open disks the sum of whose radii is not greater than $4 \eta R$, we have

$$
\ln |f(z)| \geqslant-H(\eta) \ln M(2 e R, f)
$$

for

$$
H(\eta)=2+\ln (3 e / 2 \eta)
$$

Let $\eta<1 / 8$ and choose $R$ sufficiently large so that $2+8 \eta R<R$. Using Theorem 2.2 there must exist a circle of radius $R_{1}$ with $R_{0}=(1-8 \eta) R-2 \leqslant$ $R_{1}<R$ so that the circle $|z|=R_{1}$ does not intersect any of the excluded disks and hence

$$
\left|g_{1}(z)\right| \geqslant M\left(2 e R, g_{1}\right)^{-H(\eta)}
$$

for $|z|=R_{1}$. Choose $R_{1}$ as small as possible. This implies that $R_{1} \leqslant R_{0}+$ $8 \eta R \leqslant R-2$. Now 


$$
M\left(r, g^{*}\right)=\max _{|z|=r}\left|\sum_{i_{0}+\ldots+i_{k}<n} a_{i_{0} \ldots i_{k}}^{*}(z)(f)^{i_{0}}\left(f^{\prime}\right)^{i_{1}} \ldots\left(f^{(k)}\right)^{i_{k}}\right|
$$

$$
\begin{aligned}
& \leqslant n^{k+1} \max _{i_{0}+i_{1}+\ldots+i_{k}<n} \max _{|z|=r}\left|a_{i_{0} \ldots i_{k}}^{*}(z)(f)^{i_{0}}\left(f^{\prime}\right)^{i_{1}} \ldots\left(f^{(k)}\right)^{i_{k}}\right| \\
& \leqslant c_{1} \exp \left(c_{2} r\right) \max _{i_{0}+\ldots+i_{k}<n}\left(M(r, f)^{i_{0}} M\left(r, f^{\prime}\right)^{i_{1}} \ldots M\left(r, f^{(k)}\right)^{i_{k}}\right) .
\end{aligned}
$$

2.5. LEMMA. For any nonzero entire function $f(z)$ and any $\delta>0$ we have

$$
M\left(r, f^{(K)}\right) \leqslant K ! M(r, f)^{K \delta} M\left(r+M(r, f)^{-\delta}, f\right) .
$$

Proof. We have

$$
f^{(K)}(z)=\frac{K !}{2 \pi i} \oint_{|\xi-z|=M(r, f)^{-\delta}} \frac{f(\xi)}{(\xi-z)^{K+1}} d \xi .
$$

Thus, if we choose $z$ so that $|z|=r$ and $\left|r^{(K)}(z)\right|=M\left(r, f^{(K)}\right)$ we have

$$
\begin{aligned}
M\left(r, f^{(K)}\right)=\left|f^{(K)}(z)\right| & =\left|\frac{K !}{2 \pi i} \oint_{|\xi-z|=M(r, f)^{-\delta}} \frac{f(\xi)}{(\xi-z)^{K+1}} d \xi\right| \\
& \leqslant \frac{K !}{2 \pi} \cdot 2 \pi M(r, f)^{-\delta} \frac{M\left(r+M(r, f)^{-\delta}, f\right)}{M(r, f)^{-(K+1) \delta}} \\
& =K ! M\left(r+M(r, f)^{-\delta}, f\right) M(r, f)^{K \delta} .
\end{aligned}
$$

Using the fact that in (2.4) we have $K \leqslant k$ and $i_{0}+\ldots+i_{k} \leqslant n-1$ we get from (2.6) that

$$
M\left(r, g^{*}\right)<c_{1} \exp \left(c_{2} r\right) M\left(r+M(r, f)^{-\delta}, f\right)^{n-1} M(r, f)^{k(n-1) \delta} .
$$

Since $g=g^{*} / g_{1}$ and $M\left(r, g_{1}\right)<c_{3} \exp \left(c_{4} r\right)$ we have

$$
\left|g_{1}(z)\right|^{-1} \leqslant M\left(2 e R, g_{1}\right)^{H(\eta)}<c_{5} \exp \left(c_{6} R\right)
$$

on $|z|=R_{1}$. Combining with (2.7) we get

(2.8) $M\left(R_{1}, g\right)<c_{7} \exp \left(c_{8} R\right) M\left(R_{1}+M\left(R_{1}, f\right)^{-\delta}, f\right) M\left(R_{1}, f\right)^{k(n-1) \delta}$.

If we use this estimate of $g$ in (2.1) we get

$$
M\left(R_{1}, f\right)^{n}=M\left(R_{1}, f^{n}\right) \leqslant M\left(R_{1}, h\right)+c_{9} R_{1}^{m} \exp \left(c_{10} R_{1}\right) M\left(R_{1}, g\right)
$$

$$
\leqslant c_{11} \exp \left(c_{12} R\right) M\left(R_{1}+M\left(R_{1}, f\right)^{-\delta}, f\right)^{n-1} M\left(R_{1}, f\right)^{k(n-1) \delta} .
$$


Here we used the obvious fact that $r^{m} \leqslant 2^{m r}$ and the innocuous assumption that $f$ is not identically 0 .

We now are in a position to prove Theorem 1.4 for the case $R=$ C. Assume to the contrary that $f$ is not of exponential type and pick $\delta=1 /(2 k(n-1))$. Then (2.9) becomes

$$
M\left(R_{1}, f\right)^{n-1 / 2} \leqslant c_{11} \exp \left(c_{12} R\right) M\left(R_{1}+M\left(R_{1}, f\right)^{-\delta}, f\right)^{n-1} .
$$

Now choose $R$ to be large and so that $M\left(R_{0}, f\right)>c_{11}^{4} \exp \left(4 c_{12}\left(R_{0}+1\right) /(1-8 \eta)\right)$ and hence

$$
M\left(R_{1}, f\right)>M\left(R_{0}, f\right)>c_{11}^{4} \exp \left(4 c_{12}\left(R_{0}+1\right) /(1-8 \eta)\right)=c_{11}^{4} \exp \left(4 c_{12} R\right)
$$

and in addition $M\left(R_{1}, f\right)>2^{4(n-1) / \delta}>2^{1 / \delta}$. Then $(2.10)$ yields

$$
M\left(R_{1}, f\right)^{n-3 / 4} \leqslant M\left(R_{1}+M\left(R_{1}, f\right)^{-\delta}, f\right)
$$

or

$$
\begin{aligned}
M\left(R_{1}, f\right)^{1+1 / 4(n-1)} & \leqslant M\left(R_{1}+M\left(R_{1}, f\right)^{-\delta}, f\right) \\
& \leqslant M\left(R_{1}+1 / 2, f\right) \leqslant M\left(R_{2}, f\right)
\end{aligned}
$$

where $R_{2} \geqslant R_{1}+1 / 2$ is the least radius so that the circle $|z|=R_{2}$ does not intersect any of the excluded open disks in the inequality (2.3) for $g_{1}$. Such a radius, $R_{2}<R-1$, must exist since the sum of the diameters of the excluded disks is $\leqslant 8 \eta R$.

Now using (2.7) and (2.3) for $|z|=R_{2}$ we have (2.8) with $R_{1}$ replaced by $R_{2}$ and (2.11) becomes

$$
M\left(R_{2}, f\right)^{1+1 / 4(n-1)} \leqslant M\left(R_{2}+M\left(R_{2}, f\right)^{-\delta}, f\right),
$$

and therefore

$$
\begin{aligned}
M\left(R_{1}, f\right)^{(1+1 / 4(n-1))^{2}} & \leqslant M\left(R_{2}, f\right)^{1+1 / 4(n-1)} \\
& \leqslant M\left(R_{2}+M\left(R_{2}, f\right)^{-\delta}, f\right)
\end{aligned}
$$

Thus

$$
\begin{aligned}
M\left(R_{2}, f\right)^{\delta} & \geqslant M\left(R_{1}, f\right)^{\delta(1+1 / 4(n-1))} \\
& \geqslant 2^{(4(n-1) / \delta) \delta(1+1 / 4(n-1))} \geqslant 2^{2} .
\end{aligned}
$$

Thus 


$$
M\left(R_{1}, f\right)^{(1+1 / 4(n-1))^{2}} \leqslant M\left(R_{2}+1 / 2^{2}\right) \leqslant M\left(R_{3}, f\right)
$$

where $R_{3} \geqslant R_{2}+1 / 4$ is selected in a way analogous to the method of selection for $R_{2}$. Repeating this process gives us

$$
M\left(R_{1}, f\right)^{(1+1 / 4(n-1))^{N}} \leqslant M\left(R_{N}, f\right) \leqslant M(R, f)
$$

for all $N=1,2, \ldots$ which is impossible since the left-hand side tends to infinity while the right-hand side remains bounded by $M(R, f)$.

In [2, Theorem 2.1] it was shown that, if $R_{\rho}$ is the ring of entire functions of order $\leqslant \rho$ which at the $s$ points $z_{\sigma} ; \sigma=1,2, \ldots, s$, have Gaussian integral derivatives of all orders and if $\rho<s$, then for every $t$ functions $f_{\tau} ; \tau=1, \ldots, t$, in $R_{\rho}$ with

$$
t>s\left(\left(\frac{s-1}{s-\rho}\right)^{2}-1\right)
$$

there exist linear differential operators $L_{\tau} \in \mathbf{Z}[i, D]$ with Gaussian integral coefficients not all 0 such that

$$
\sum_{\tau=1}^{t} L_{\tau} f_{\tau}=0
$$

Thus, if $f \in R_{\rho}$ we can apply (2.16) to the functions $f^{\tau} ; \tau=1, \ldots, t$, and obtain a differential equation

$$
\sum_{\tau=1}^{t} L_{\tau} f^{\tau}=0
$$

If $n$ is the maximal index in (2.17) for which $L_{n} \neq 0$ we get

$$
L_{n} f^{n}=-\sum_{v=1}^{n-1} L_{v} f^{v}
$$

which is an equation of type (1.1) with $R=\mathbf{C}$.

Hence, by what we have just proved, the function $f$ must be of bounded exponential type.

2.19. THEOREM. An entire function which together with all its derivatives has Gaussian integral values at s points is either of bounded exponential type or of order $\rho \geqslant s$.

As was shown in [2] this gap in the order of such integral valued functions is the correct gap in the sense that for each $\rho \geqslant s$ the set of functions in $R_{\rho}$ of order $\rho$ is of the power $2^{\aleph} 0$ while, for certain choices of $z_{1}, \ldots, z_{s}$, 
there do exist functions of bounded exponential type which are infinitely Gaussian integral valued at these points. Combining Theorem 2.19 with Theorem 2.1 of [2] we get

2.20. THEOREM. If $\rho<s$ then every function of order $\rho$ which has Gaussian integral derivatives of all orders at $s$ points is the solution of a linear differential equation with Gaussian integral coefficients, and hence is either an exponential polynomial or a polynomial.

If we restrict attention to rationally integral valued functions, we get, from $[2, \S 4]$,

2.21. THEOREM. If $s>1$ and there exists a nonconstant entire function $f(z)$ of order $\rho<s$ which has rationally integral derivatives of all orders at the points $z_{1}=0, z_{2}, \ldots, z_{s}$ then there are only the following possibilities:

1. $z_{2}, \ldots, z_{s}$ are rational and $f(z)$ is a polynomial with rational coefficients.

2. $z_{2}, \ldots, z_{s}$ are logarithms of rational numbers and $f(z)$ is a polynomial with rational coefficients in $e^{z}$ and $e^{-z}$.

3. $z_{2}, \ldots, z_{s}$ are of the form $\left(\cosh ^{-1} \sqrt{1+d r^{2}}\right) / \sqrt{d}$ where $r$ is rational and $\sqrt{1+d r^{2}}$ is rational. Here $d$ is a (square free) integer and $f(z)$ is a polynomial with rational coefficients in $\cosh \sqrt{d z}$ and $(\sinh \sqrt{d z}) / \sqrt{d}$.

It is easy to see that there are functions of the form $A z, A e^{z}$ and $A$ $\cosh \sqrt{d z}$ which are infinitely integral valued at $z_{1}, \ldots, z_{s}$ in cases 1,2 and 3 respectively.

Theorem 2.21 is interesting in showing a type of analytic dependence of the points $z_{1}, \ldots, z_{s}$ which in a natural way generalizes the property of belonging to a fixed algebraic number field. We could have obtained very similar results with restrictions of values to elements of a general algebraic number field $K$. For general $K$ we will need as many analytic functions as we have Archimedean valuations on $K$ and we defer the details for a later paper. The case in which $K$ has only one Archimedean valuation, that is an imaginary quadratic number field, can be settled by the methods used in [2] for $Q(i)$. In the case $K=Q(i)$ Theorem 2.21 becomes

2.22. THEOREM. If $s>1$ and there exists a nonconstant entire function $f(z)$ of order $\rho<s$ which has Gaussian integral derivatives of all orders at the points $z_{1}=0, z_{2}, \ldots, z_{s}$ then there are only the following possibilities:

1. $z_{2}, \ldots, z_{s} \in Q(i)$ and $f(z) \in Q(i)[z]$.

2. $z_{2}, \ldots, z_{s}$ are logarithms of Gaussian rationals and $f(z) \in Q(i)\left[e^{z}, e^{-z}\right]$. 
3. $i z_{2}, \ldots, i z_{s}$ are logarithms of Gaussian rationals and $f(z) \in$ $Q(i)\left[e^{i z}, e^{-i z}\right]$.

4. $z_{2}, \ldots, z_{s}$ are of the form $\left(\cosh ^{-1} \sqrt{1+d r^{2}}\right) / \sqrt{d}$ where $r \in Q(i)$, $\sqrt{1+d r^{2}} \in Q(i), d \in Z(i), d$ square free in $Z(i)$, and

$$
f(z) \in Q(i)[\cosh \sqrt{d z}, \sqrt{d} \sinh \sqrt{d z}] .
$$

It may be worthwhile to contrast these results with those of E. Bombieri [1], where the differential ring of meromorphic functions of several variables is postulated to be finitely generated over an algebraic number field $K$ and to contain transcendental functions. Then, under suitable growth conditions of the functions, it is possible to deduce conditions on the points at which the functions assume values in $K$.

In our investigation we start from functions satisfying growth conditions and arithmetic conditions at certain points and derive the property that their ring is differential and finitely generated from those conditions.

3. The case $R=\mathrm{C}[z]$. We shall once again set $g=P\left(f, f^{\prime}, \ldots, f^{(k)}\right)$ in (1.1) and if we consider (1.1) to be a nonhomogeneous differential equation with polynomial coefficients we can replace it by the system of equations $w_{1}=$ $f^{n}, w_{1}^{\prime}=w_{2}, w_{2}^{\prime}=w_{3}, \ldots, w_{m-1}^{\prime}=w_{m}, C_{m} w_{m}^{\prime}=g-C_{0} w_{1}-\ldots-$ $C_{m-1} w_{m}$, which gives us the matrix equation

$$
\vec{w}^{\prime}=A(z) \vec{w}+B(z)
$$

where

$$
A(z)=\left(\begin{array}{ccccc}
\vec{w}=\left(w_{1} \ldots w_{m-1}\right. & \left.w_{m}\right)^{T}, \\
0 & 1 & 0 & \ldots & 0 \\
\vdots & 0 & 1 & \ldots & 0 \\
-\frac{C_{0}}{C_{m}} & -\frac{C_{1}}{C_{m}} & -\frac{C_{2}}{C_{m}}- & \ldots & -\frac{C_{m-1}}{C_{m}}
\end{array}\right),
$$

where ${ }^{T}$ denotes transpose. If $C_{m}$ is a constant we may choose $C_{m}=1$, if not then $\left|C_{m}(z)\right| \geqslant 1, z$ sufficiently large. In either case there exists an $a$ so that $\left|C_{m}(z)\right| \geqslant 1$ for $|z| \geqslant a$. Now for each $|z|>a$ we pick a path $P(z)$ consisting of the $\operatorname{arc} a e^{i \theta}, 0 \leqslant \theta \leqslant \arg z$, followed by the segment $t z, a /|z| \leqslant t \leqslant 1$. In order to estimate the magnitude of the solutions of (4.1) we apply the succes- 
sive approximations procedure

$$
\vec{\phi}_{k+1}(z)=\vec{v}+\int_{a}^{z} A(t) \vec{\phi}_{k}(t) d t+\int_{a}^{2} \vec{B}(t) d t
$$

where $\vec{v}$ is a constant vector and integration is along $P(z)$.

If we let $\phi_{0}=0$ then

$$
\vec{\phi}_{1}(z)-\vec{\phi}_{0}(z)=\vec{\phi}_{1}(z)=\vec{v}+\int_{a}^{z} \vec{B}(t) d t
$$

and if, for any matrix function $E(t)$, we let the maximum value of the norm, $|E(t)|$, of $E(\tau)$ as $\tau$ varies over $P(t)$ be $M(t, E)$ we have

$$
\begin{aligned}
\left|\vec{\phi}_{2}-\vec{\phi}_{1}\right| & =\left|\int_{a}^{z} A(t)\left(\vec{\phi}_{1}(t)-\vec{\phi}_{0}(t)\right) d t\right| \leqslant \int_{a}^{z} M(t, a) M\left(t, \vec{\varphi}_{1}\right)|d t| \\
& \leqslant M(z, A) M\left(z, \vec{\varphi}_{1}\right) \int_{a}^{z}|d t| \leqslant M(z, A) M\left(z, \vec{\varphi}_{1}\right) l(z)
\end{aligned}
$$

where $l(z)$ is the length of the path $P(z)$ and the norm used on vectors and matrices is the $L_{1}$-norm.

Now

$$
\begin{aligned}
\left|\vec{\phi}_{3}-\vec{\phi}_{2}\right| & =\left|\int_{a}^{z} A(t)\left(\vec{\phi}_{2}(t)-\phi_{1}(t)\right) d t\right| \\
& \leqslant \int_{a}^{z}|A(t)|\left|\vec{\phi}_{2}(t)-\vec{\phi}_{1}(t)\right| d t\left|\leqslant \int_{a}^{2} M(t, A)^{2} M\left(t, \vec{\varphi}_{1}\right) l(t)\right| d t \mid \\
& <M(z, A)^{2} M\left(z, \vec{\varphi}_{1}\right) \int_{a}^{2} l(t) d l(t)=M(z, A)^{2} M\left(z, \vec{\varphi}_{1}\right) l^{2}(z) / 2 ! .
\end{aligned}
$$

Iterating this method we get

$$
\left|\vec{\phi}_{k+1}-\vec{\phi}_{k}\right| \leqslant M\left(z, \vec{\varphi}_{1}\right)(l(z) M(z, A))^{k} / k !
$$

We write

$$
\begin{aligned}
\left|\vec{\phi}_{j}(z)\right| & =\left|\sum_{k=0}^{j-1}\left(\vec{\phi}_{k+1}(z)-\vec{\phi}_{k}(z)\right)\right| \\
& \leqslant M\left(z, \vec{\varphi}_{1}\right) \sum_{k=0}^{j-1} \frac{(l(z) M(z, A))^{k}}{k !}<M\left(z, \vec{\varphi}_{1}\right) \exp (l(z) M(z, A)) .
\end{aligned}
$$

We let $N(r, E)=\max _{|z|=r} M(z, E)$ and we have

$$
\left|\vec{\phi}_{j}(z)\right|<N\left(r, \vec{\varphi}_{1}\right) \exp (2 r N(r, A))
$$


where $r=|z|$. Assume $r>6 a$ so that $l(z) \leqslant 2 \pi a+r-a<2 r$. It follows then that

$$
|\vec{w}(z)| \leqslant N\left(r, \vec{\varphi}_{1}\right) \exp (2 r N(r, A))
$$

where $\vec{w}$ is a solution vector for (4.1) and therefore

$$
M(r, f)^{n}=M\left(r, f^{n}\right) \leqslant N\left(r, \vec{\varphi}_{1}\right) \exp (2 r N(r, A)) .
$$

Now we can choose $M_{R}=M_{\mathrm{C}[z]}$ to be the semiring of functions $M(r)$ of the form $M(r)=c r^{p} ; c, p \geqslant 0, p$ an integer. The corresponding $M_{F}$ is

$$
M_{F}=\left\{\sum_{i=1}^{N} c_{i 1} \exp \left(c_{i 2} r^{p}\right) \mid c_{i j}>0, p \geqslant 1, p \text { an integer }\right\} .
$$

If we repeat the argument and the values $R_{0}, R_{1}$ and $R$ used in $\S 2$ to obtain (2.8) with the upper bounds chosen from $M_{F}$ above we get the inequality

$$
M\left(R_{1}, g\right)<c_{1} \exp \left(c_{2} R^{p}\right) M\left(R_{1}+M\left(R_{1}, f\right)^{-\delta}, f\right)^{n-1} M\left(R_{1}, f\right)^{k(n-1) \delta} .
$$

Since $|\vec{B}(z)|=\left|g(z) / C_{m}(z)\right|$ and $\left|C_{m}(z)\right| \geqslant 1$ if $|z|=r \geqslant a$ we have $M\left(z, \vec{\varphi}_{1}\right)$ $<c_{3} r M(r, g) ;|z|=r$. This gives us $N\left(r, \vec{\varphi}_{1}\right)<c_{3} r M(r, g)$. We also have $|A(z)|<c_{4} r^{q}$ if $r \geqslant a$ and therefore $N(r, A)<c_{4} r^{q}$ so that (4.2) becomes

$$
M\left(R_{1}, f\right)^{n} \leqslant c_{5} \exp \left(c_{6} R^{j}\right) M\left(R_{1}+M\left(R_{1}, f\right)^{-\delta}, f\right)^{n-1} M\left(R_{1}, f\right)^{k(n-1) \delta}
$$

where $j$ is an integer $\geqslant 1$, and we have again used the fact that $r \leqslant 2^{r}$ and the assumption that $f$ is not identically zero.

We can now prove Theorem 1.4 for the case $R=\mathbf{C}[z]$. Assume that $f(z)$ is not of finite order. Choose $\delta=1 /(2 k(n-1))$ so that (3.4) becomes

$$
M\left(R_{1}, f\right)^{n-1 / 2} \leqslant c_{7} \exp \left(c_{8} R^{j}\right) M\left(R_{1}+M\left(R_{1}, f\right)^{-\delta}, f\right)^{n-1} .
$$

Now if $f$ is not of finite order we choose a large $R$ so that the corresponding $R_{0}=(1-8 \eta) R-2$ satisfies

$$
M\left(R_{1}, f\right) \geqslant M\left(R_{0}, f\right) \geqslant c_{7}^{4} \exp \left(4 c_{8}\left(R_{0}+2\right)^{j} /(1-8 \eta)^{j}\right)=c_{7}^{4} \exp \left(4 c_{8} R^{j}\right)
$$

and at the same time

$$
M\left(R_{1}, f\right)>2^{4(n-1) \delta}>2^{1 / \delta} .
$$

As in $\$ 2$ we now get a sequence of radii $R_{1}<R_{2}<\ldots<R$ so that $R_{i}+1 / 2^{i} \leqslant R_{i+1}$ and

$$
M\left(R_{1}, f\right)^{(1+1 / 4(n-1))^{N}} \leqslant M\left(R_{N}, f\right)<M(R, f)
$$

for all $N=1,2, \ldots$, a contradiction. 
Note that our method has in fact proved that the order of $f$ is no more than the greater of the maximum of the orders of the coefficients of $g=$ $P\left(f, f^{\prime}, \ldots, f^{(k)}\right)$ and $1+$ the maximum of the degrees of $C_{0}, \ldots, C_{m-1}$.

4. Concluding remarks. The results in Theorem 1.4 can be extended to general entire coefficients $C_{i}(z)$ and polynomials $P\left(f, f^{\prime}, \ldots, f^{(k)}\right)$ with entire coefficients as long as $C_{m}$ has only a finite number of zeros in order to yield upper bounds of the form $M_{F}$.

For general entire $C_{m}(z)$ and general meromorphic coefficients of the polynomial $P$ the lower bound estimates needed on $C_{m}^{-1}$ and of the denominator of the coefficients of $P$ lead to new complications and the necessity for delicate estimates which we hope to give in a subsequent paper.

The results in [4] have been extended to rings of meromorphic functions of a non-Archimedean variable [5]. The more general theory developed here should have analogous generalization most likely with analogously sharper results.

\section{BIBLIOGRAPHY}

1. E. Bombieri, Algebraic values of meromorphic maps, Invent. Math. 10 (1970), 267-287. MR 46 \#5328.

2. A. H. Cayford, $A$ class of integer valued entire functions, Trans. Amer. Math. Soc. 141 (1969), 415-432. MR 39 \#5800.

3. B. Ja. Levin, Distribution of zeros of entire functions, GITTL, Moscow, 1956; English transl., Transl. Math. Monographs, vol. 5, Amer. Math. Soc., Providence, R. I., 1964. MR 19, 402; 28 \#217.

4. E. G. Straus, Differential rings of meromorphic functions, Acta Arith. 21 (1972), 271-284. MR 46 \#7532.

5. - Differential rings of analytic functions of a nonarchimedean variable, Diophantine Approximation and its Applications, Academic Press, New York, 1973, pp. 295308.

DEPARTMENT OF MATHEMATICS, UNIVERSITY OF BRITISH COLUMBIA, VANCOUVER, BRITISH COLUMBIA, CANADA

DEPARTMENT OF MATHEMATICS, UNIVERSITY OF CALIFORNIA, LOS ANGELES, CALIFORNIA 90024 